\title{
Symptomatic treatment of unresponsive wakefulness syndrome with transcranially focused extracorporeal shock waves
}

\author{
H. Lohse-Busch*, U. Reime and R. Falland \\ Outpatient Department for Manual Medicine - Movement Disorder Center, Rheintalklinik, Bad Krozingen, \\ Germany
}

\begin{abstract}
.
OBJECTIVE: Can stimulation of nerve growth factors by focused transcranial extracorporeal shock wave therapy (TESWT) be made effective for persons within unresponsive wakefulness syndrome (apallic syndrome)?

DESIGN: Between eight and 18 years after the brain lesion, five patients with unresponsive wakefulness syndrome of differing severity received TESWT with the device Duolith (Storz Medical) during four-week physicomedical complex therapies. In the previous years they had been treated with the same complex therapies without TESWT. The vigilance did not change.

RESULTS: After two-four years and an average of 5.2 treatment series, the patients' abilities improved by $135.9 \%$ on the German Coma Remission Scale (KRS) and by $43.6 \%$ on the Glasgow Coma Scale. In the motor area of the KRS, the patients improved by $64.3 \%$. Three PEG feeding tubes could be removed, nonverbal communication initiated four times.

CONCLUSION: In this longitudinal observation study, focused TESWT stimulated vigilance in patients with unresponsive wakefulness syndrome. The precise neurophysiological effects remain to be verified by a study of the clinical results.
\end{abstract}

Keywords: Unresponsive wakefulness syndrome, apallic, extracorporeal shock wave therapy

\section{Introduction}

Differential diagnostic classification of states of severe limitations of consciousness is difficult. The causes include hypoxia following drug abuse, states following inflammatory brain diseases, tumor diseases, trauma sequelae and intoxications. The results are variously described by the terms coma vigile, apallic syndrome, vegetative state, disconnection syndrome and persistent vegetative state. The diversity of the damaging influences accounts for the qualitative and topographical variety of their effects on the brain. This

\footnotetext{
*Address for correspondence: H. Lohse-Busch, MD, Outpatient Department for Manual Medicine - Movement Disorder Center, Rheintalklinik, Im Rheintal 5, D-79189 Bad Krozingen, Germany. Tel.: +49 7633408 836; Fax: +49 7633408 842; E-mail: lohsebusch@amm-rheintalklinik.de.
}

state of affairs leads to a differentiation in the degree of vigilance in the affected patients from coma to states of minimal consciousness to less severe disorders of consciousness. We thus prefer to speak of the state as "unresponsive wakefulness syndrome" (Laureys, 2010), as suggested by the European Task Force on Disorders of Consciousness.

The physicomedical and medication treatment of unresponsive wakefulness syndrome is restricted to adequate muscle and joint care, stimulation in the somatic and cognitive area and reduction of the commonly pronounced spasticity. The expectation of improvement in vigilance diminishes with increasing time intervals following the onset of brain damage.

The results of the therapy with focused low-energy extracorporeal shock waves (ESWT) of patients with spastic disorders (Amelio, 2010; Manganotti, 2005; 
Lohse-Busch, 1997; Lohse-Busch, 2010) suggest that this form of therapy not only acts on the muscles, but also stimulates neural structures.

It is now known that it stimulates the production of endothelial nitrogen oxides (eNOs) (Mariotto 2009) and (via the endothelial vascular growth factor (VEGF)) angiogenesis (Ito, 2009) and neurogenesis (Sun, 2006). Neurogenesis is also promoted by FGF-2 (fibroblast growth factor) (Laird, 1995; Mudò, 2009), which is also stimulated by ESW. Lymphangiogenesis is positively influenced via the stimulation of endothelial lymph vessel growth factor (eLGF) (Kubo, 2010). Following application of ESW to a hip necrosis, a systemic increase in the concentration of NO, VEGF, and FGF was detectable in serum over a period of four weeks, and is no longer significantly elevated after 3 months (Wang, 2011). In the animal model, VEGF promotes functional regeneration and slows down secondary degeneration after experimental contusions of the spinal cord (Widenfalk, 2003).

In this context, the working hypothesis has been put forward that extracorporeal shock waves may restore synaptic connections in the brain that once existed but are now malfunctioning.An attempt was therefore made to stimulate the central nervous system in patients with unresponsive wakefulness syndrome with focused transcranial ESWT (TESWT). The investigation was planned as a longitudinal observation study.

\section{Methods}

The Freiburg University Hospital Ethics Commission announced that it had no objections to the performance of the treatments.

The parents of the patients were informed in detail about the experimental character of the treatments, both in writing and orally. Despite the possible risks of adverse effects, the carers declared their consent in writing for the treatments planned.

\subsection{Inclusion criteria}

The patients had to have been suffering from a stable unresponsive wakefulness syndrome over a period of least 5 years, so that appreciable improvements in the vigilance sector were no longer to be expected.

\subsection{Exclusion criteria}

Increase in any negative symptoms in the area of motor function or vigilance during the treatment period.
Epileptic seizures during the treatment period. Changes of antispasmodic or antiepileptic medication during the observation period. Changes of domestic carers. Parental decision to discontinue treatment.

\subsection{Treated patients}

During the years 2005-2012, five patients who had suffered brain damage between eight and 18 years prior to the treatment series with TESWT and who met the inclusion criteria were examined and treated, four men and one woman, with an average age of 38.6 years (28-45 years). One patient had experienced prolonged hypoxia during a narcotic coma following heroin abuse; the other four patients had suffered severe skull injuries in traffic accidents. All patients had spastic tetraparesis of differing severity. In three patients, soft tissue release operations on the muscles of the upper and lower extremities had been performed to improve body care and positioning. Three patients suffered from epilepsy subsequent to their craniocerebral traumas that is reliably controlled with medication. Four patients were fed via a PEG feeding tube.

\subsection{Basic complex therapy}

All of the patients had received a wide variety of forms of rehabilitation during the years following brain damage. Their medical histories were very well known as they had all been treated up to eight times for fourweek periods by our treatment team in previous years, for an average of 5.2 times.

The multimodal complex treatment performed during these periods comprised a workday, intensive serial treatment with different forms of manual medicine, physiotherapy on a neurophysiological basis, the use of medicomechanic devices, ergotherapy and ESWT on the spastically movement-impaired extremities three times a week. The therapeutic goal was in each case adequate muscle and joint care. With these treatments, it was possible to achieve a reduction in spastic dystonia and muscular rigidity. This facilitated patient body care, but no improvement in vigilance occurred.

\subsection{Additional TESWT}

In addition to the unchanged complex treatment described above, each of the five patients received 4000 focused extracorporeal shock waves three times a week over a period of four weeks with an energy flow density of $0.20 \mathrm{~mJ} / \mathrm{mm}^{2}$ to $0.30 \mathrm{~mJ} / \mathrm{mm}^{2}$ and a length of focus 
Table 1

Table 1 German KRS, and equivalent of the international CRS*. The six readily comparable items yield a maximum of 24 points on the German KRS and 23 points on the CRS

\begin{tabular}{|c|c|c|}
\hline & KRS max. points & Revised JFK CRS max. points \\
\hline 1. Arousal/Attentiveness & 5 & 3 \\
\hline \multicolumn{3}{|l|}{ Arousal scale* } \\
\hline 2. Motor function response & 6 & 6 \\
\hline \multicolumn{3}{|l|}{ Motor function scale $*$} \\
\hline 3. Reaction to acoustic stimulus & 3 & 4 \\
\hline \multicolumn{3}{|l|}{ Auditory function scale* } \\
\hline 4. Reaction to visual stimulus & 4 & 5 \\
\hline \multicolumn{3}{|l|}{ Visual function scale* } \\
\hline 5. Reaction to tactile stimulus in German KRS only & 3 & Equivalent in arousal scale \\
\hline 6. Oromotor response & 3 & 3 \\
\hline \multicolumn{3}{|l|}{ Oromotor/verbal function scale* } \\
\hline 7. Communication scale $*$ & $\begin{array}{l}\text { With small difference equivalent in } \\
\text { 1. Arousal/Attentiveness }\end{array}$ & 2 \\
\hline Maximum point score & 24 & 23 \\
\hline
\end{tabular}

Table 2

Presentation of the results after an average of 5.2 treatment series with the complex treatment including TESWT, beginning an average of 11.2 years after the cerebrocranial trauma

\begin{tabular}{|c|c|c|c|c|c|c|c|c|c|c|}
\hline Patient no. & Age & $\begin{array}{l}\text { n Series } \\
\text { with } \\
\text { ESWT } \\
\text { only }\end{array}$ & $\begin{array}{l}\text { Onset of the } \\
\text { ESW n } \\
\text { years after } \\
\text { accident }\end{array}$ & $\begin{array}{l}\text { Onset of } \\
\text { TESWT } \\
\text { n years } \\
\text { after } \\
\text { accident }\end{array}$ & $\begin{array}{l}\text { n Series } \\
\text { with } \\
\text { TESWT }\end{array}$ & $\begin{array}{c}\text { TESWT } \\
\text { over } n \\
\text { years }\end{array}$ & $\begin{array}{l}\text { KRS before } \\
\text { TESWT } \\
\text { max. point } \\
\text { score } 24\end{array}$ & $\begin{array}{r}\text { KRS after } \\
\text { TESWT }\end{array}$ & $\begin{array}{l}\text { GCS before } \\
\text { TESWT } \\
\text { max. point } \\
\text { score } 15\end{array}$ & $\begin{array}{r}\text { GCS after } \\
\text { TESWT }\end{array}$ \\
\hline 1 & 45 & 1 & 17 & 18 & 4 & 4 & 5 & 17 & 4 & 10 \\
\hline 2 & 39 & 6 & 8 & 11 & 8 & 3 & 6 & 16 & 6 & 10 \\
\hline 3 & 28 & 5 & 7 & 9 & 5 & 2,5 & 7 & 19 & 9 & 11 \\
\hline 4 & 45 & 2 & 7 & 8 & 4 & 2 & 9 & 23 & 11 & 12 \\
\hline 5 & 36 & 8 & 6 & 10 & 5 & 2,5 & 12 & 17 & 9 & 13 \\
\hline Aver-age & 38.6 & 4.4 & 9 & 11.2 & 5.2 & 2.8 & 7.8 & 18.4 & 7.8 & 11.2 \\
\hline Changes in $\%$ & & & & & & & & $135.90 \%$ & & $43.60 \%$ \\
\hline
\end{tabular}

of $5.5 \mathrm{~cm}$ to the neurocranium. For safety reasons and to spread the shock waves as widely as possible during the treatments, the therapy source and hence the focus were kept in continuous motion. The TESWT was conducted using the device Duolith (Storz Medical).

Patient no. 1 was treated once per year over a period of four weeks with TESWT; patients nos. 2-4 underwent four-week treatments approximately every six months. The average observation period for the treatment series with TESWT was 2.4 years.

\subsection{Assessments}

At the beginning of the treatments with TESWT, the abilities of the patients were measured both by the physicians as well as physiotherapists using the German Coma Remission Scale (KRS) (Lippert-Grüner, 2002) and the Glasgow Coma Scale (Jennett, 1975).

The Glasgow Coma Scale is a very rough measuring instrument which is, however, the one most commonly used internationally. However, only very rough indi- cations are provided on the development of a coma in the presence of chronic unresponsive wakefulness syndrome.

The German Coma Remission Scale (KRS) is used for differential evaluation of patients with severe disturbances of consciousness in German-speaking countries. It has received very positive evaluations and is recommended by the German "Neurological \& Neurosurgical Early Rehabilitation Working Group". In Germanspeaking countries, it is the most commonly used scoring system for disturbances of consciousness in patients with protracted coma remission. Both qualitatively as well as quantitatively, it is very similar to the revised JFK Coma Recovery Scale (CRS) (Kalmar, 2005) that is used in the English-speaking parts of the world. There are only very small differences in the study protocol itself and the weighting of the abilities, as can be seen in Table 1 .

The KRS is used to measure the disturbance of consciousness and is much more differentiated than the GCS in its investigation of wakeability, attentiveness 
Table 3

Presentation of the point scores for motor responses only

\begin{tabular}{lcc}
\hline Patient no. & $\begin{array}{c}\text { KRS motor Response } \\
\text { before TESWT }\end{array}$ & $\begin{array}{c}\text { KRS motor response } \\
\text { after TESWT }\end{array}$ \\
\hline 1 & 2 & 4 \\
2 & 2 & 4 \\
3 & 3 & 5 \\
4 & 3 & 5 \\
5 & 4 & 5 \\
Average & 2,8 & 4,6 \\
Change in \% & & $64.3 \%$ \\
\hline
\end{tabular}

KRS before TESWT

- KRS after TESWT

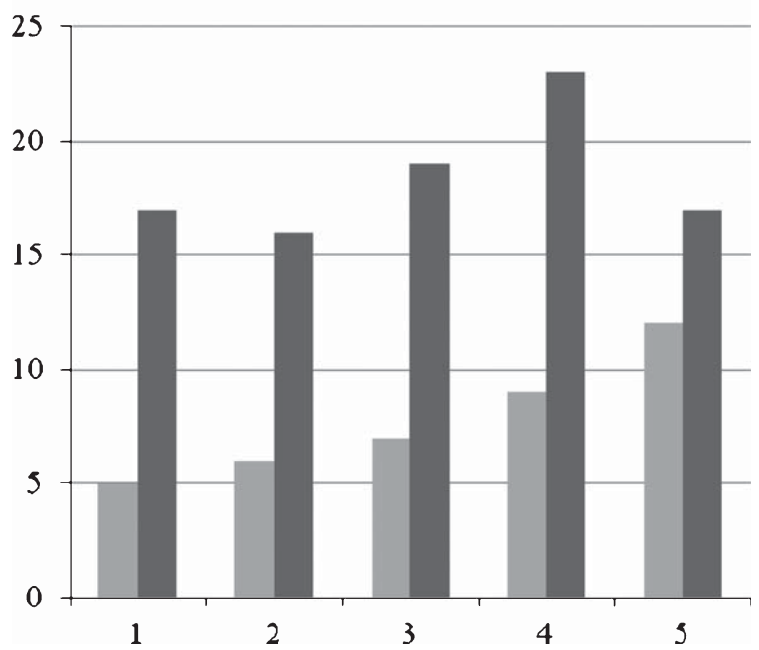

Fig. 1. Presentation of the point scores on the KRS as a result of treatment with TESWT in the five patients.

and the best motor and oromotor function response to different stimuli. Furthermore, self-initiative and the ability to communicate are measured much effectively than is the case with the GCS.

After the treatment series, the point scores of the KRS and GCS were determined once again. The assessments of the patients' abilities by the physiotherapists were approximately $10 \%$ higher in each case than those made by the physicians. Tables 2 and 3 show the lower point scores determined by the physicians.

\section{Results}

There was no change in vigilance among the patients in previous years after they were given an average of 4.4 treatment series with ESWT on muscles of the extremities.
KRS mot before TESWT

KRS mot after TESWT

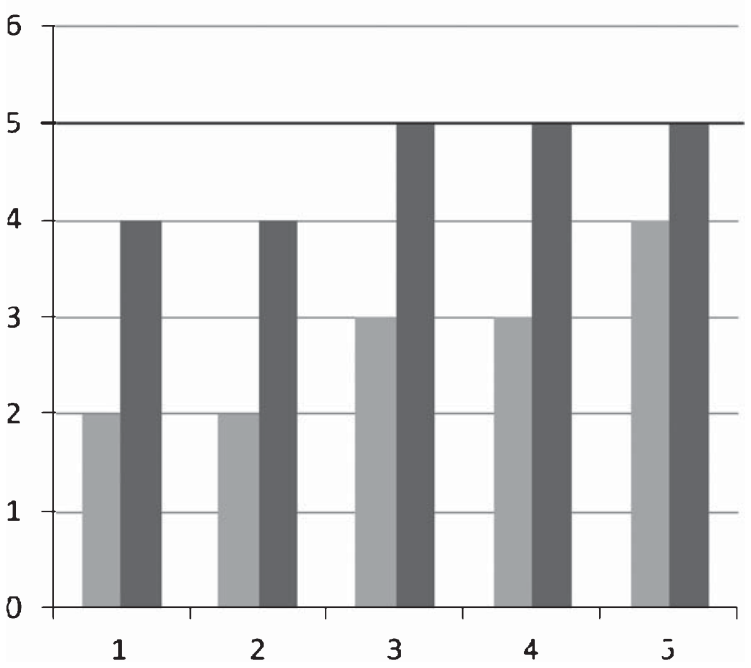

Fig. 2. Separate consideration of the motor responses in tetraparetic patients.

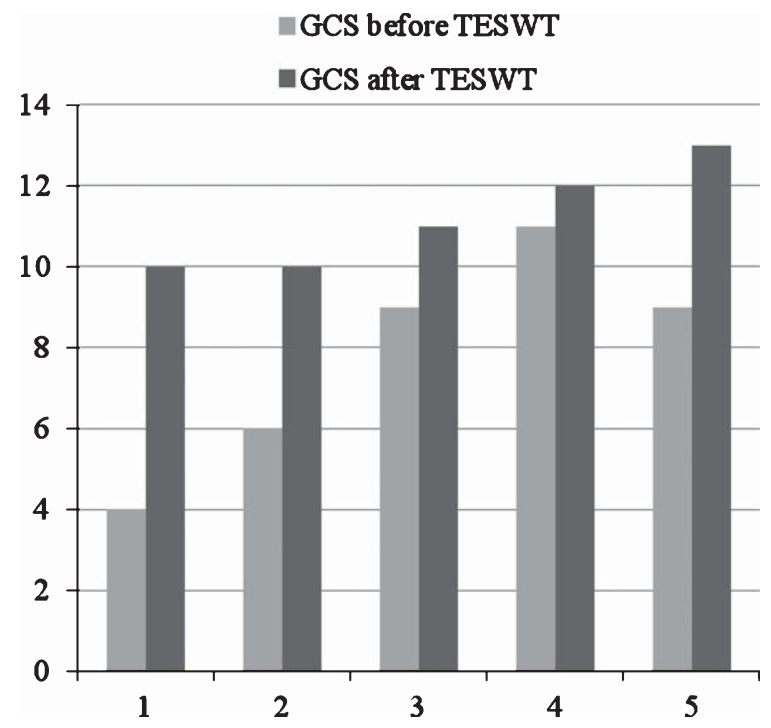

Fig. 3. Presentation of point scores on the GCS before and after TESWT.

After 4-8, (average of 4.5) treatment series over 2.8 years with TESWT, there is an average increase in the point score in the KRS of $135.9 \%$ (Table 2, Fig. 1).

Motor responses in accordance with the guidelines of the KRS Test Manual are not to be evaluated in tetraparetic patients. However, because motor abilities also improved during the observation period, the 
maximum point scores were nevertheless set at 24 for the consideration of the results.

A separate consideration of the motor performance of the tetraparetic patients in temporal relationship with the increase in vigilance showed an improvement of $64.3 \%$ (Table 3, Fig. 2).

There were no adverse effects, in particular not the triggering of epileptic fits.

There is an increase on the GCS of $43.6 \%$. Accordingly, the three abilities assessed do not adequately reflect the improvement in the patients' vigilance in comparison with the KRS (Table 2, Fig. 3).

\subsection{Casuistry}

\subsubsection{Patient 1}

The patient suffered a traffic accident at the age of 23 . In addition to severe cerebrocranial trauma, there were fractures of the right thigh and the os pubis on the right, and ruptures of the spleen and liver. The patient suffered multiple intracranial hemorrhages with large tissue defects in the temporal region on both sides, resulting in a midbrain syndrome with dextral tetraspasticity and a disconnection syndrome. There was maximum extensor spasticity of the lower extremities and flexor spasticity of the upper extremities with extreme muscular rigidity. A focal seizure disorder was well-controlled with medication. The patient had a PEG feeding tube because he was not able to chew or to swallow liquids. There was no control over salivary flow. Emptying of the bladder was ensured by catheterization. The patient wore diapers because of fecal incontinence.

Seven years after the accident, because of spastic deformities and in order to facilitate body care, the flexor tendons of all of the toes, the knee flexors, the quadriceps and the gluteus maximus were each severed on both sides, and achillotenotomies were performed, as were a release of the right shoulder and of the right elbow.

The patient did not open his eyes in response to a pain stimulus but responded with extensor synergies only. There was no random motor activity whatsoever and no facial expression. No vocal sounds or moaning could be provoked.

There was no control over the head. Despite fixation with a head band to the headrest of the wheelchair, the head leaned continually to the left; due to an eyelid ptosis, the right eye was almost always closed. The left eye had a fixed gaze to the right.

Seventeen years after the accident the patient was given the complex treatment described, which was con- ducted over a period of three weeks. He achieved 5 points on the KRS and 4 points on the GCS. During the treatment series three times a week, focused ESW was applied to the muscles of the extremities to reduce muscular stiffness and hypertonia. The goal of facilitating body care was achieved. There were no changes beyond this.

Eighteen years after the accident, the complex treatment was supplemented by TESWT three times per week. At yearly intervals the patient received a total of four treatment series of four weeks' duration. After four years, continually increasing his score, he reached 17 points on the KRS and 10 points on the GCS.

It became possible to hold a proper conversation using different communicative techniques for more than half an hour. Facial expression has increased. The patient recognizes people, welcomes them with a smile and follows persons walking around the room with his eyes. Upon request, when looking at a picture, he is able to direct his gaze to specific parts of the picture. In addition to the blinking code, smiling for agreement and growling for rejection are now at his disposal. If the patient wishes to attract attention, there are rudimentary vocalizations. A buzzer can also be used specifically by retroversion of the right arm for a yes/no code.

Rudimentary eye-hand control can be seen when the patient uses an intentional movement of his shoulder to touch the strings of a guitar held in front of him with his hand. With respect to motor function, it may be further mentioned that the patient is better able to expectorate. Three-dimensional control of the head has not improved, although he requires less supportive assistance from the physiotherapist when standing in the standing exerciser. It appears he uses his extensor spasticity intentionally in supported standing.

The achievements described are very greatly dependent on daily form.

\subsubsection{Patient 2}

After an overdose of heroin at the age of 28 , the patient fell into a narcotic coma and suffered severe cerebral hypoxia. The comatose state under intensive medical care lasted for six months. Unresponsive wakefulness syndrome and tetraparesis with maximum extensor spasticity of the lower as well as maximum flexor spasticity of the upper extremities developed. Despite antiepileptic medication, two-three times per year the patient has a grand mal seizure lasting two-five minutes.

Four years after the accident, to facilitate body care, tenotomies were performed on the plantar flexors, knee flexors, flexors of the elbows and the finger flexor 
tendons. Due to the very restricted ability to chew and especially to swallow liquids, the patient was fed via a PEG feeding tube. The overflow bladder is to be catheterized. The patient is provided with diapers.

The patient came to our clinic eight years after the onset of brain damage. There were no intentional movements of the extremities or of the spine. The head was held rigidly rotated to the left and bent forward. If the head was fixated with a head band, the eyes turned upon request, although with a delay. The gaze remained rigid, however. The facial expression was also rigid and generally showed a depressive expression.

The response to pain stimuli was an unarticulated growling, after which the facial expression changed for a few seconds to an expression of uncontrolled anger. Salivary flow was not under control.

The patient could be brought passively into the vertical position in the standing exerciser, extensor spasticity holding him upright.

Initially, over a period of three years he received fourweek complex treatments each year with the application of focused ESW three times per week to the muscles of the extremities. In this way, muscular hypertonicity and rigidity could be markedly reduced, facilitating body care.

Eleven years after the accident, application of TESWT was begun in addition to the known complex treatments. Previously the patient scored 6 points on the KRS and 6 points on the GCS. Over a period of four and a half years, a total of eight complex treatments with TESWT of four weeks' duration in each case were performed.

After the fourth series, the PEG feeding tube was removed because the patient was able to chew and also to swallow liquids. There was considerably better control of salivary flow. No voice-like utterances were observed.

At the end of the eighth series, the patient achieved 16 points on the KRS and 10 points on the GCS. He recognized people and welcomed them with a smile. They were fixed with a gaze and followed around the room with an adequate turning of the head. The facial expression was no longer depressive but definitively changed to friendly and amused phases.

In physiotherapy sessions, over the course of time the patient was increasingly able to help with position transfer accomplishments. He turned upon request from the lateral to the prone position with little help.

The patient answers correctly to questions with a blurred yes or no. Other words are "mum" and "enough". These, however, are used only very rarely.
A yes/no code was developed using movements. A lifting of the right shoulder means yes, movement of the left thumb means no. The patient opens his eyes for more than 15 minutes at a time and is awake and attentive when doing so. He enjoys pressing an electric button with his head to switch a radio on and off upon request. These achievements were very dependent on daily form. Hardly any attentiveness could be achieved in the late hours of the afternoon.

In the evening hours, the patient followed television broadcasts for more than 30 minutes and laughed at jokes at the right times.

\subsubsection{Patient 3}

The patient suffered a traffic accident at the age of 19. Diffuse cerebral hemorrhages occurred. Large tissue defects remain in the brainstem region, temporooccipital right and frontal left. The posttraumatic coma lasted 10 days. In addition, the patient had fractures of the sternum, right clavicle and the right upper ankle joint.

Six weeks after the accident the thoracic aorta was dissected, accompanied by massive bleeding. An unresponsive wakefulness syndrome and tetraspastic movement disorder emerged with maximum extensor spasticity of the lower extremities and maximum flexor spasticity of the upper extremities as well as the correspondingly typical contractures. The application of intrathecal baclofen via a pump ameliorated the spasticity somewhat.

Due to inadequate control of oral motor functions and the pharyngeal musculature, the patient was fed via a PEG feeding tube. He voided urine via a suprapubic catheter and was provided with diapers.

There was no control over the head. When the patient was sitting, the head fell forwards in an uncontrolled manner onto the chest. In the area of the upper extremities, intentional, if albeit dystonic movements of the shoulder joints in response to pain were possible but not upon command. The elbow joints and hands could not be moved intentionally.

The patient was able to express contentment and discontent using facial expression and unarticulated vocalizations. A yes/no code was not possible because the patient was unable to make such decisions. The attention span was less than five seconds, the patient opening his eyes and smiling. Apart from this, however, he was apathetic and exhibited a rigid facial expression. He occasionally looked at someone coincidentally but without fixing his eyes on pictures or people; there was no response to painless, tactile stimuli. 
The patient came under our treatment seven years after his accident; before admission into TESWT, he had received complex treatments of four weeks' duration including ESWT on the musculature of the extremities over a period of two years for a total of four times each. These treatments markedly reduced both the rigidity and the spastic hypertonia, so that body care and hence quality of life was improved for a period of about four months.

In addition, the patient received TESWT for the first time nine years after the accident. Prior to this, he had reached 7 points on the KRS and 9 points on the GCS. Over a period of two and a half years, a total of five four-week complex treatments with supplementary TESWT were performed. After this treatment, the patient achieved 19 points on the KRS and 11 points on the GCS.

After the third treatment series with TESWT, the PEG feeding tube was able to be removed because oropharyngeal motor function enabled eating and also the swallowing of liquids. Attentiveness could be maintained without any effort for the duration of a physiotherapeutic or ergotherapeutic therapy session lasting 45 minutes.

The spontaneous reactions of facial expression take place immediately. The patient uses differentiated vocalization to indicate whether he likes or dislikes something. A yes/no code was able to be developed with the help of a buzzer. "No" is expressed by shaking the head to the right. He laughs a lot, behaving appropriately to the situation when he does so, and shows that he very much likes singing. It is clearly apparent that he can remember amusing situations from earlier times. When he is reminded of these, he smiles.

Control over the head has increased to the extent that people fixed with the eyes can be followed through the room by turning the head held upright.

Upon request both upper extremities are moved from the shoulder separately. The left thumb is used specifically to press the keys of a keyboard. When standing under assistance, there is adequate control of the head. When walking in a walking device with a saddle to sit on, the lower extremities are placed forward in alternation by the patient himself.

The achievements described are independent of daily form and can be demonstrated at any time.

\subsubsection{Patient 4}

When the patient was 40 years old, he suffered a cerebrocranial trauma with considerable lacunar bleeding as the result of a traffic accident. The patient suffered contusions with tissue defects in the right temporooccipital and temporofrontal region in the hippocampus and parahippocampus region, in the corpus callosum, the left pyramidal tract and in the brainstem. In addition, there were serial fractures to ribs $4-6$ on the right, luxation of the right elbow joint, and fracture of the ossa pubis on both sides. After neurosurgical and surgical treatment the patient was initially placed into an artificial coma that was terminated after two weeks.

A spastic hemiparesis remained on the right with considerable dystonia and general motor ataxia, aphasia with a possibly remaining understanding of words and a pronounced retardation of psychomotor function. Four years after the trauma, the patient achieved 9 points on the KRS and 11 points on the GCS.

The patient held his head bent forward and turned to the left. The facial expression was generally rigid and was similar to a facies leontina. A smile would appear in each case after several seconds' delay, as when welcoming someone, for example, when the patient was in a phase of relative wakefulness. He chewed and also swallowed liquids.

The attention span was approximately 20 seconds. The patient was able to ward off painful stimuli specifically with his hands. He did not, however, use his hands spontaneously or upon verbal request. He recognized familiar voices and fixed his eyes on pictures and people, but he did not follow them with his eyes around the room. Vocalizations comprised growling and groaning.

Four years after the accident, the patient received two series of the physicomedical complex treatment, each lasting four weeks and including ESWT, for the spastic mobility disturbances to the muscles of the extremities. A marked reduction in rigidity and spastic hypertonia was able to be achieved this way which lasted for three to four months.

The described complex treatments with the inclusion of TESWT were begun five years after the accident. The patient received two such treatment series, each of four weeks' duration, with a six-month interval in between. Afterwards, he achieved 23 points on the KRS and 12 points on the GCS.

All cognitive processes now proceed with only a very small short. The attention span is approximately $30 \mathrm{~min}-$ utes. The patient makes eye contact, and also follows people in the room with his eyes and by turning his head with increasingly improved head control. The patient grasps a rod with one or both hands upon request, bending and stretching his elbows when doing so, and can release the rod once again upon request. The application of force with the hands can be adequately varied. 
The beginning of communication has been initiated by closing his eyes or pressing the buzzer once to mean "yes". Expressing "no" by pressing the buzzer twice has not yet been reliably achieved. No vocalization has been achieved.

All achievements have developed continuously over the period of a year, but they are highly dependent on daily form. On some days, the patient's attention cannot be aroused at all for periods of several hours.

\subsubsection{Patient 5}

When she was 26 years old, the patient suffered a motorcycle accident with a fracture of the fifth thoracic vertebra and a cerebrocranial trauma, resulting in complete flaccid paraparesis (ASIA A) below TH 5. Surgical treatment of the thoracic vertebral fracture was followed by pneumonia, which ultimately made intubation with machine-supported ventilation necessary. This was accompanied by hypoxidosis, which meant that the patient suffered from diffuse, hypoxic brain damage. This resulted in cognitive and psychical retardation with very slowed reactions and maximum spastic-hypertonic paresis of the upper extremities.

Different intensive rehabilitation measures carried out twice a year, each time for a duration of four weeks, ultimately resulted in no further changes of the condition after two years.

A PEG feeding tube was installed because the patient could not swallow, even though she could chew. Salivary flow was not under control. Evacuation of the bladder was via suprapubic catheter. Because of fecal incontinence, the patient was provided with diapers.

The attention span was about two minutes. The patient gave the impression of being very slowed down in terms of cognitive performance. Her only means of communication was to express annoyance, pain, etc. by unarticulated vocalization, screaming or moaning. Persons were followed around the room with the eyes. No other intentional motor functions were possible, apart from turning the head from the midline to the left, chewing and moving the eyes.

The patient came to our clinic six years after the accident and in the succeeding years initially received the described complex treatment of four weeks' duration at intervals of six months each. This involved ESWT three times per week in the region of the extremities to ameliorate muscular stiffness and hypertonia, thus considerably facilitating body care. During these years, the patient achieved an unchanged 12 points on the KRS and 9 points on the GCS.
In the tenth year after the accident, in addition to the complex treatment being continued unchanged the patient received each time three TESWT per week. Over a period of two and a half years, the patient received five treatment series of four weeks' duration at half-yearly intervals.

After the second treatment series with TESWT, the PEG feeding tube was able to be removed as swallowing could now be performed without any problems. Continuous positive development occurred during this period, so that the patient ultimately achieved 17 points on the coma remission scale and 13 points on the GCS.

The patient is able to cooperate with complete concentration for the duration of a physiotherapy or ergotherapy therapy session, greets people with a friendly smile, is amused by jokes and can use the words yes and no absolutely appropriately, without the previous delay in reaction times.

Upon request, she can also follow people in the room by turning her head over the midline by $40^{\circ}$, both to the right and to the left. The right shoulder can be used reliably upon request for the buzzer code or switching on/off of a radio. Salivary flow outside mealtimes is very well controlled.

The achievements could as a general rule be observed at any time throughout the entire day. Only in times of great tiredness does the patient sink back once again into a semi-conscious state for one to two hours.

\section{Discussion}

The effect of one form of energy or another striking living tissue is dependent on the strength and length of exposure. High-energy ESW can demyelinate nerves (Wu, 2008) and destroy brain tissue (Lee, 2007; Kato, 2007). Doing this requires either very high energy in a single pulse (Kato, 2007) or several hundred pulses applied punctually to the same treatment site a number of times (Wu, 2008; Lee, 2007). To avoid the possibility of damage to nerve structure due to accumulation of ESW at a small area of tissue during the treatment of the patients described here, an energy flow density at the focus of $0.15 \mathrm{~mJ} / \mathrm{mm}^{2}$, in exceptional cases $0.2 \mathrm{~mJ} / \mathrm{mm}^{2}$, is not exceeded and the therapy source is moved continuously.

It must be assumed that the skull absorbs the majority of the energy from the TESWT. It is not known how much energy actually reaches the brain tissue. It could be approximately $10 \%$. Accordingly, it may be assumed that the energy flow density at the focus which 
actually reaches the brain can be estimated to be in the range of $0.01 \mathrm{~mJ} / \mathrm{mm}^{2}-0.02 \mathrm{~mJ} / \mathrm{mm}^{2}$. The relatively low energy flow density and the constantly moved therapy source did not cause any adverse effects or painful irritations whatsoever to develop. In the self-experiment conducted by the first author of this study named, it was possible to determine that treatments with a moving focus within the cited energy flow density are not painful.

Low-energy ESW do not damage the spinal nociceptive system (Haake, 2002) and can contribute to the repair of damaged structures of the medulla (Sun, 2006; Widenfalk, 2003) by activating the neurotrophic system. This takes place by means of the shock waveinduced, local and systemic increase in the eNOs (Mariotto, 2009), the VEGF (Ito, 2009) and the FGF (Laird, 1995; Hausdorf, 2011). The VEGF facilitates the recovery of a spinal cord injury (Liu, 2010) and stimulates the growth of neurites in the rat (Jin, 2006) and the mouse models (Sun, 2006).

The hypothesis of the single stimulation limited temporally to the treatment system does not, however, explain the protracted course of the improvements. The effect of the treatment with TESWT follows a development with a tendency towards improvements in the second half of the four-week treatment series. This improvement then continues for about a month and proves useful in the domestic environment. None of the patients has lost his or her newly regained abilities in the interval of six to twelve months before the next treatment series.

It seems conceivable that structures experience repair that takes time. This phenomenon could be explained by the fact that the local and systemic increases in the concentrations of NO, of the VEGF, and the FGF are detectable over a period of about four weeks (Wang, 2011) and then gradually fall again. In one portion of the patients described here, an increase in the symptomatic improvements were noticed over this period of time. More clarity must be established here in subsequent studies.

Our patients had suffered their severe to most severe disturbances of consciousness between seven and eighteen years before the application of TESWT and for many years had experienced no changes in their vigilance. All the patients profited from the transcranial treatment with focused extracorporeal shock waves in that their vigilance underwent considerable improvement. In 4 patients, nonverbal communication was able to be initiated for the first time since the onset of brain damage, even though its performance was dependent on the daily form. The PEG feeding tube was able to be removed from three of the patients. Despite the small number of patients, it can be noted that those who are especially severely handicapped profit most from the treatment.

All patients give the impression that they enjoy the increase in vigilance. This is especially apparent during physiotherapy and ergotherapy when they are able to achieve things that were not previously possible.

Even during observation periods of many years duration, our treatments have never evoked any adverse effects whatsoever. The treatment technique is evidently safe.

Questions concerning the optimum treatment rhythm and optimum dosage of TESWT must remain open because this study is comprised only of preliminary observations. The possibilities at a rehabilitation clinic are limited. It would be a welcome development if neurological centers, which have more extensive laboratory procedures at their disposal, were able to address this subject.

\section{Conclusion}

In this longitudinal observation study, focused TESWT stimulated vigilance in 5 patients with unresponsive wakefulness syndrome. TESWT may therefore be a new non-invasive option for alleviating the difficult-to-improve symptoms of these brain damage sequelae.

\section{Declaration of interest}

The corresponding author received a research grant from Storz Medical AG, Taegerwilen, Switzerland.

\section{Acknowledgments}

The non-profit association "Mobilitas e. V." funded the assessments and the publication fee.

\section{References}

Amelio, E., \& Manganotti, P. (2010) Effect of shock wave stimulation on hypertonic plantar flexor muscles in patients with cerebral palsy: A placebo-controlled study. J Rehabil Med, 42, 339-343.

Haake, M., Thon, A., \& Bette, M. (2002) No influence of low-energy extracorporeal shock wave therapy (ESWT) on spinal nociceptive systems. J Orthop Sci, 7, 97-101. 
Hausdorf, J., Sievers, B., Schmitt-Sody, M., Jansson, V., Maier, M., \& Mayer-Wagner, S. (2011) Stimulation of bone growth factor synthesis in human osteoblasts and fibroblasts after extracorporeal shock wave application. Arch Orthop Trauma Surg, 131, 303-309.

Ito, K., Fukumoto, Y., \& Shimokawa, H. (2009) Extracorporeal shock wave therapy as a new and non-invasive angiogenic strategy. Tohoku J Exp Med, 219, 1-9.

Jennett, B., \& Bond, M. (1975) Assessment of outcome after severe brain damage. Lancet, 1480-1484. 1-23.

Jin, K., Mao, X. O., \& Greenberg, D. A. (2006) Vascular endothelial growth factor stimulates neurite outgrowth from cerebral cortical neurons via Rho kinase signaling. J Neurobiol, 66, 236-242.

Kalmar, K., \& Giacino, J. T. (2005) The JFK coma recovery scalerevised. Neuropsychol Rehabil, 15, 454-460.

Kato, K., Fujimura, M., Nakagawa, A., Saito, A., Ohki, T., Takayama, K., \& Tominaga, T. (2007) Pressure-dependent effect of shock waves on rat brain: Induction of neuronal apoptosis mediated by a caspase-dependent pathway. J Neurosurg, 106, 667-76.

Kubo. M., Li, T. S., Kamota, T., Ohshima, M., Shirasawa, B., \& Hamano, K. (2010) Extracorporeal shock wave therapy ameliorates secondary lymphedema by promoting lymphangiogenesis. J Vasc Surg, 52, 429-434.

Laird, J. M., Mason, G. S., Thomas, K. A., Hargreaves, R. J., \& Hill, R. G. (1995) Acidic fibroblast growth factor stimulates motor and sensory axon regeneration after sciatic nerve crush in the rat. Neuroscience, 65, 209-216.

Laureys, S., Celesia, G. G., Cohadon, F., Lavrijsen, J., León-Carrión, J., Sannita, W. G., Sazbon, L., Schmutzhard, E., von Wild, K. R., Zeman, A., Dolce, G.; \& European Task Force on Disorders of Consciousness (2010). Unresponsive wakefulness syndrome: A new name for the vegetative state or apallic syndrome. BMC Med, 8, 68. doi: 10.1186/1741-7015-8-68

Lee, T. C., Huang, H. Y., Yang, Y. L., Hung, K. S., Cheng, C. H., Chang, N. K., Chung, Y. H., Hu, M. S., \& Wang, C. J. (2007) Vulnerability of the spinal cord to injury from extracorporeal shock waves in rabbits. J Clin Neurosci, 14, 873-78.

Lippert-Grüner, M., Wedekind, C., Ernestus, R. I., \& Klug, N. (2002) Early rehabilitative concepts in therapy of the comatose brain injured patients. Acta Neurochir Suppl, 79, 2.
Liu, Y., Figley, S., Spratt, S. K., Lee, G., Ando, D., Surosky, R., Fehlings, M. G. (2010) An engineered transcription factor which activates VEGF-A enhances recovery after spinal cord injury. Neurobiol Dis, 37, 384-393.

Lohse-Busch, H., Kraemer, M., \& Reime, U. (1997) A pilot investigation into the effects of extracorporeal shock waves on muscular dysfunction in children with spastic movement disorders. Schmerz, 11, 108-112.

Lohse-Busch, H., Kraemer, M., Reime, U., Marbler, X., \& Möwis, U. (2010) Changes of walking abilities of children and teenagers affected by spastic cerebral palsy. A 2 week complex therapy with physical treatements. Manuelle Medizin, 48, 107-111.

Manganotti, P., \& Amelio, E. (2005) Long-term effect of shock wave therapy on upper limb hypertonia in patients affected by stroke. Stroke, 36, 1967-1971.

Mariotto, S., de Prati1, A. C., Cavalieri, E., Amelio, E., Marlinghaus, E., \& Suzuki, H. (2009) Extracorporeal shock wave therapy in inflammatory diseases: Molecular mechanism that triggers antiinflammatory action. Curr Med Chem, 16, 2366-2372.

Mudò, G., Bonomo, A., Di Liberto, V., Frinchi, M., Fuxe, K., \& Belluardo, N. (2009) The FGF- 2/FGFRs neurotrophic system promotes neurogenesis in the adult brain. J Neural Transm, 116, 995-1005.

Sun, Y., Jin, K., Childs, J. T., Xie, L., Mao, X. O., \& Greenberg, D. A. (2006) Vascular endothelial growth factor-B (VEGFB) stimulates neurogenesis: Evidence from knockout mice and growth factor administration. Dev Biol, 289, 329-335.

Wang, C. J., Yang, Y. J., \& Huang, C. C. (2011) The effects of shockwave on systemic concentrations of nitric oxide level, angiogenesis and osteogenesis factors in hip necrosis. Rheumatol Int, 31, 871-877.

Widenfalk, J., Lipson, A., Jubran, M., Hofstetter, C., Ebendal, T., Cao, Y., \& Olson, L. (2003) Vascular endothelial growth factor improves functional outcome and decreases secondary degeneration in experimental spinal cord contusion injury. Neuroscience, 120, 951-960.

Wu, Y. H., Liang, H. W., Chen, W. S., Lai, J. S., Luh, J. J., \& Chong, F. C. (2008) Electrophysiological and functional effects of shock waves on the sciatic nerve of rats. Ultrasound Med Biol, 34, 16881696. 